\title{
Should God believe the Liar? A non-dialetheist paraconsistent approach to God's Omniscience*
}

\author{
Guilherme Araújo Cardoso ${ }^{1}$ \\ http://orcid.org/0000-0003-0045-8508 \\ ${ }^{1}$ Universidade Federal de Ouro Preto \\ Department of Philosophy \\ Ouro Preto, $M G$, Brazil \\ guilherme.cardoso@ufop.edu.br
}

\section{Sérgio Ricardo Neves de Miranda ${ }^{2}$ \\ https://orcid.org/0000-0002-0192-7372 \\ ${ }^{2}$ Universidade Federal de Ouro Preto \\ Department of Philosophy \\ Ouro Preto, MG, Brazil \\ sergiornmiranda@ufop.edu.br}

\section{Article Info \\ CDD: 111.1}

Received:05.07.2021; Revised: 29.10.2021; Accepted:

04.11.2021

https://doi.org/10.1590/0100-6045.2021.V44N4.GC

Keywords

Paradoxes

Omnscience

Truth

Dialetheism

Situation Semantics

${ }^{*}$ The authors acknowledge support from the John Templeton Foundation, research grant 61556 . 


\begin{abstract}
In this paper, we discuss a family of arguments that show the inconsistency of the concept of omniscience, which is one of the central attributes of the theistic God. We introduce three member of this family: Grim's Divine Liar Paradox, Milne's Paradox and our own Divine Curry. They can be seen as theological counterparts of well-known semantic paradoxes. We argue that the very simple dialetheist response to these paradoxes doesn't work well and then introduce our own response based on a framework that we call Logic of Impossible Truths (LIT). LIT is a non-dialetheist paraconsistent logic designed to represent divine ominiscience and to preserve the transparency of the truth predicate and which semantics rests on the concept of situation. Since some rules of classical logic are not valid in LIT, we are in a position to block the derivation of the paradoxes. Thus, LIT offers a way out of the dilemma of accepting that there are true contradictions (dialetheism) or giving up the idea that there is an all-powerful, omniscient and perfectly good being (atheism).
\end{abstract}

\title{
1 Introduction
}

Theism is a theological theory that has at its core the idea that God is an omnipotent, omniscient and perfectly good being, who creates the world and everything else in it. Although the theist concept of God has been widely accepted by believers of many stripes, including Christians, Muslims and Jews, there are powerful arguments pointing out contradictions in theism. We call them arguments of impossibility, since they show that the theist concept of God is incoherent and cannot be satisfied by any natural or supernatural entity.

The most popular argument of impossibility in the history of philosophy is the well-known Paradox of the Stone, which aims to show the incoherence of the divine attribute of omnipotence by asking whether 
God can create a stone so heavy that he cannot lift. The arguments of impossibility we discuss in this article are not so famous as the Paradox of the Stone, but we think that they are so interesting and powerful as that argument and even harder to solve.

Grim (Grim, 1991) was the first to call attention to a family of arguments that jeopardize the coherence of the attribute of divine omniscience. We are especially interested in the argument he dubbed as the Divine Liar. In this paper, we present Grim's Divine Liar argument and show that it is actually a recipe to build a family of paradoxes. We call them the Liars. We introduce then a new member of this family: the Divine Curry. Next we show that Cotnoir's (Cotnoir, 2017) dialetheist response to the Liars' family is not adequate, because it weakens the connection between factivity, trust and divine omniscience. Our own response hangs on the semantic theory LIT we develop in section 4 of this article. We show that LIT is an appropriate logic for theism that blocks of the destructive power of the Liars. Finally, we draw some lessons from adopting LIT as a framework for the solution of paradoxes.

\section{Paradoxes}

The common understanding of the concept of omniscience is that an omniscient being knows everything, which means that this being knows the truth-values of every sentence or proposition about the past, present and future, no matter its content, as well the truth of eternal sentences, e.g. theorems of logic and mathematics. It also belongs to the set of basic intuitions concerning the concept of knowledge that the person who knows $x$ is in a better position to provide information about $x$ and make correct judgements on this matter. We then say that this 
person is reliable, that we can trust what she says. Likewise, an omniscient being must be seen as an absolutely reliable being, we must absolutely trust his words no matter what. The all encompassing character of God's knowledge and the absolute reliability of God's judgements are captured in the following definition of omniscience:

Definition 1. (Omniscience) A being $x$ is omniscient iff for all sentence $\alpha$, (i) $\alpha$ is true iff $x$ believes that $\alpha$, (ii) $\alpha$ is false iff $x$ doesn't believe that $\alpha$, (iii) if $\alpha$ were true, then $x$ would believe that $\alpha$, and finally (iv) if $\alpha$ were false, then $x$ would not believe that $\alpha$.

Conditions (i) and (ii) are individually necessary for omniscience. Condition (i) is necessary because it excludes from the set of omniscient things that being who has very few beliefs or has true beliefs of a single type only (e. g. this being has some true beliefs based on perception and doesn't form beliefs by reasoning or any other belief forming process). An omniscient being not only has true beliefs, but must have an infinite number of true beliefs of every kind imaginable. Condition (ii) is also necessary for omniscience, since it excludes from the set of omniscient things that being who is capable of thinking every conceivable sentence, but who also accepts everything that comes to her mind as being true. The point here is not only that an omniscient being must be able to correctly separate the set of sentences into two subsets, the one to which only the true sentences belong and the other to which only the false sentences belong, but also that the difference between believing the truth and only the truth and believing falsehoods really matters. In this way, an omniscient being will have a very exclusive set of beliefs, inasmuch as no falsehood is allowed in this set. 
Conditions (iii) and (iv) are also necessary. First consider a person who has a true belief that $\alpha$ in a situation $s_{1}$, but would maintain her belief in a new situation $s_{2}$ similar to $s_{1}$, except that $\alpha$ is false in $s_{2}$. Even if this person has actually only true beliefs, she doesn't look like an omniscient being. After all, she could easily make mistakes in her judgements. Consider now a person who would change her belief that $\alpha$ because of insignificant changes in her environment that are unrelated to the truth of $\alpha$. We certainly wouldn't say that she is omniscient, because she also could easily fall in error. In short, a being who is not able of tracking the truth of sentences in different situations doesn't deserve to be called omniscient. Conditions (iii) and (iv) perfectly capture this important tracking aspect of the omniscience.

Conditions (i)-(iv) capture also the idea of an omniscient being as absolutely trustful. First consider a central function of knowledge attributions: when we say that a person knows that $\alpha$, we single her out as a good informant, we distinguish her as someone capable of giving a reliable information about a topic that concerns us. As Craig (Craig, 1990) puts it briefly, "the concept of knowledge is used to flag approved sources of information". It looks like that we can say something similar about the attributions of omniscience. An omniscient being is an absolutely trustful source of information, which means that such a being has not only a maximal stock of truths at her disposal, but also that this being is not prone to error and track all truths in a reliable and infallible way. We can put it briefly in this way: to say that God is omniscient is also to say that we trust him and are ready to act accordingly to his words.

We assume that conditions (i)-(iv) are together sufficient for the attribution of omniscience and that our analysis is correct. So we can now state 
important rules related to the concept of omniscience. Let $B$ represent the belief relation, $\mathfrak{g}$ an individual constant that names God, and the formula $B\left(\mathfrak{g},{ }^{r} \alpha^{\urcorner}\right)$ the sentence that God believes that $\alpha$ is the case. We have then the following rules for omniscience:

$$
\begin{array}{ll}
\text { (Capture) } & \alpha \vDash B\left(\mathfrak{g},{ }^{\ulcorner} \alpha^{\urcorner}\right) \\
\left(\text {Capture }^{*}\right) & \neg \alpha \vDash \neg B\left(\mathfrak{g},{ }^{\ulcorner} \alpha^{\urcorner}\right) \\
(\text {Release }) & \left.B\left(\mathfrak{g},{ }^{\ulcorner} \alpha\right\urcorner\right) \vDash \alpha \\
\text { Release } \left.^{*}\right) & \neg B\left(\mathfrak{g},{ }^{\ulcorner} \alpha^{\urcorner}\right) \vDash \neg \alpha
\end{array}
$$

Capture says that if $\alpha$ is the case, then we can infer that God believes that $\alpha$, and Release says if God believes that $\alpha$, it follows that it is the case that $\alpha$. Capture $^{*}$ and Release* incorporate negation to these rules. So Capture* says that if it is not the case that $\alpha$, then we can infer that God doesn't believe that $\alpha$, and Release* says that if God doesn't believe that $\alpha$, then it follows that it is not the case that $\alpha$. We think that conditions (iii) and (iv) would also support more complex rules for the omniscience operator. But for now, we deal only with Capture, Release, Capture* and Release* rules, because they are enough to build the paradoxical arguments that are the focus of this article.

The starting point of Grim's argument is the sentence of the Divine Liar:

$$
\text { God believes that }(\mathfrak{d} \mathfrak{l}) \text { is false }
$$

By the Principle of Bivalence (PB), $(\mathfrak{d} \mathfrak{l})$ is true or false. On the one hand, if $(\mathfrak{d} l)$ is true, it follows by Capture that God believes that (ol), i.e. God believes that God believes that (ol) is false. Release says that 
every sentence that God believes holds and so we have: God believes that $(\mathfrak{d} \mathfrak{l})$ is false. We get now a problem. If $(\mathfrak{d} \mathfrak{l})$ is true, God believes that $(\mathfrak{d} \mathfrak{l})$ is false. Since God believes that $(\mathfrak{d} \mathfrak{l})$ is false while $(\mathfrak{d} \mathfrak{l})$ is in fact true, God believes a falsity. Therefore, if $\mathfrak{d} l$ is true, God is not omniscient by the condition (ii) of the above definition. On the other hand, if $(\mathfrak{d} \mathfrak{l})$ is false, then it follows by Capture *that God doesn't believe that ( $\mathfrak{d} \mathfrak{l})$, i.e. God doesn't believe that God believes that (ol) is false. By Release*, it follows that it is not the case that God believes that $(\mathfrak{d} l)$ is false. In this way, given the assumption that $(\mathfrak{d} \mathfrak{l})$ is false, if God doesn't believe that $(\mathfrak{d} \mathfrak{l})$ is false, then God doesn't believe the true sentence that says that $(\mathfrak{d} \mathfrak{l})$ is false. Therefore, if $(\mathfrak{d} \mathfrak{l})$ is false, God isn't omniscient by condition (i) of the above definition. Since it follows from the truth of $(\mathfrak{d} l)$ and also from the falsity of ( $\mathfrak{d} l)$ that God isn't omniscient, we conclude that God by no means is omniscient.

Grim develops his objection without appealing to the rules Capture, Release, Capture* and Release*, but his argument is essentially the same as the argument of the last paragraph. The following version of the Divine Liar argument is a bit different from Grim's argument and establishes the explicit contradiction that $\mathfrak{o l}$ is true and false at the same time. Assume that in the language we are talking about God's attributes we have a predicate that is had by a sentence if and only if this sentence is not believed by God, and that we also have resources to build a self-referential sentence that says of itself that it has this predicate. Thus we have a sentence, $\mathfrak{d} l$, such that, $\mathfrak{d} \mathfrak{l} \leftrightarrow \quad \neg B\left(\mathfrak{g},{ }^{\mathfrak{d}} \mathfrak{l}^{\mathfrak{l}}\right) !^{1} \quad$ Given these

\footnotetext{
${ }^{1}$ To present the informal version of Divine Liar as it is given by Grim, we use ( $\mathfrak{d} \mathfrak{l})$ as naming the sentence $\mathfrak{d}$ l. From now on, we name this sentence with ' $\mathfrak{d} \mathfrak{l}$. In arithmetical theories, corner quotes are used as numerals for Gödel-codings of formulas. Arithmetization of syntax allows obtaining self-reference in a strong and formal way. We also use corner quotes as naming
} 
assumptions, the argument is the following:
1. $\mathfrak{d} \mathfrak{l} \leftrightarrow \neg B\left(\mathfrak{g},{ }^{\mathfrak{d}} \mathfrak{d} \mathfrak{l}^{\urcorner}\right)$
2. $\mathfrak{d} \mathfrak{l} \vee \neg \mathfrak{d} \mathfrak{l}$

3. $\mathfrak{d l}$

4. $\neg B\left(\mathfrak{g},{ }^{\mathfrak{\gamma}} \mathfrak{d} \mathfrak{l}^{\urcorner}\right)$

5. $\neg \mathfrak{d} \mathfrak{l}$

6. $\mathfrak{d} \mathfrak{l} \wedge \neg \mathfrak{d} \mathfrak{l}$

7. $\neg \mathfrak{d} \mathfrak{l}$

8. $\neg B\left(\mathfrak{g},{ }^{\mathfrak{r}} \mathfrak{d} \mathfrak{l}\right)$

9. $\mathfrak{d l}$

10. $\mathfrak{d} \mathfrak{l} \wedge \neg \mathfrak{d} \mathfrak{l}$

11. $\mathfrak{d} \mathfrak{l} \wedge \neg \mathfrak{d} \mathfrak{l}$
[Self-Reference]

[LEM]

[Hypothesis]

[1,3, and $\mathrm{MP}]$

$\left[4\right.$, Release $\left.{ }^{*}\right]$

$\left[3,5\right.$, and $\left.\wedge^{+}\right]$

[Hypothesis]

$\left[7\right.$, Capture $\left.^{*}\right]$

[1,8, and MP]

$\left[7,9\right.$, and $\left.\wedge^{+}\right]$

$\left[2-10\right.$, and $\left.\vee^{-}\right]$

Semantic paradoxes are endless resources for creating problems for the concept of omniscience, as it is shown by Grim's argument and our own version based on Self-Reference and the rules of Capture, Release, Capture*, and Release*2 As it should be clear by now, the paradoxical sentence $\mathfrak{d} l$ is a theological version of the very well known Liar Sentence, a sentence that says of itself it is not true, with the only difference between the two that in the

functions, but we are not restricted to arithmetical language and arithmetical theories, since we are trying to grasp aspects of truth and God's omniscience that appears in more inclusive fragments of natural language.

${ }^{2}$ The reader might check that a contradiction follows from this equivalence even in an intuitionistic logic, in which LEM and Classical Reductio Ad Absurdum (RAA: $\neg \alpha \rightarrow \alpha \vDash \alpha$ ) do not hold, but a special version of RAA (RAAI: $\alpha \rightarrow \neg \alpha \vDash \neg \alpha$ ) and MP hold. 
Liar Sentence the truth predicate takes the place of the omniscience predicate. Truth also delivers analogous rules like Capture, Capture*, Release, and Release $^{*}$ that are based on an important feature of the concept of truth, its Transparency. Thus we have a sentence $\mathfrak{l}$, such that, $\mathfrak{l} \leftrightarrow \neg T\left({ }^{\mathfrak{r}} \mathfrak{l}\right)$, and a very similar argument to the one given above shows that a contradiction follows from the existence of $\mathfrak{l}$ and Transparency.

In fact, we can create another interesting paradox of omniscience based on the so called Curry paradox. The trouble-making sentence of the Curry paradox is a sentence $\mathfrak{c}$ that is equivalent to a conditional that has $T\left({ }^{r} \mathfrak{c}^{\urcorner}\right)$itself as antecedent and an arbitrary sentence $\alpha$ as a consequent. From self-reference, there is a sentence $\mathfrak{c}$, such that $\mathfrak{c} \leftrightarrow\left(T\left({ }^{r} \mathfrak{c}^{\urcorner}\right) \rightarrow \alpha\right)$. From Transparency and substitution of equivalents, $\mathfrak{c} \leftrightarrow(\mathfrak{c} \rightarrow \alpha)$. This is a big trouble for paraconsistent theories of Truth, since the argument we can now build threatens of triviality any theory of truth in which Transparency, MP, and Contraction (or Conditional Proof - CP) hold $]^{3}$ We are going to argue that this problem shows up again in the discussion of the divine Curry paradox.

In order to state this theological version of the Curry paradox, assume again that in the language we are talking about God's attributes (our theological theory of theism $\mathcal{T}$ ) we have resources to build a self-referential sentence. In this way, we have the following sentence of the Divine Curry paradox:

If God believes $(\mathfrak{d} \mathfrak{c})$, then God is Brazilian

Thus we have a sentence $\mathfrak{d} c$, such that,

\footnotetext{
${ }^{3}$ Contraction is the rule $\Gamma, \alpha \rightarrow(\alpha \rightarrow \beta) \vDash \alpha \rightarrow \beta$. Conditional Proof $(\mathrm{CP})$ is the property of $\vDash$ according to which, if $\Gamma, \alpha \vDash \beta$, then $\Gamma \vDash \alpha \rightarrow \beta$. From either Contraction or CP, MP, and $\mathfrak{c} \leftrightarrow(\mathfrak{c} \rightarrow \alpha)$, we can prove $\alpha$, whatever sentence we replace for $\alpha$.
} 
$\mathfrak{d} \mathfrak{c} \leftrightarrow\left(B\left(\mathfrak{g},{ }^{\ulcorner} \mathfrak{d} \mathfrak{c}^{\urcorner}\right) \rightarrow \mathfrak{t}\right)$, where $\mathfrak{t}$ translates "God is Brazilian". If we have this sentence in our theory $\mathcal{T}$, then we can prove not only that God is Brazilian, but also that God isn't Brazilian and any other sentence we like. In short, our talk about God would be trivial, since everything would be provable in $\mathcal{T}$. We reason as follows:

1. $\mathfrak{d} \mathfrak{c} \leftrightarrow\left(B\left(\mathfrak{g},{ }^{\mathfrak{}} \mathfrak{d} \mathfrak{c} \mathfrak{c}^{\urcorner}\right) \rightarrow \mathfrak{t}\right)$

[Self-Reference]

2. $B\left(\mathfrak{g},{ }^{\ulcorner} \mathfrak{d} \mathfrak{c}^{\urcorner}\right) \leftrightarrow\left(B\left(\mathfrak{g}, \mathfrak{}^{\mathfrak{d}} \mathfrak{c}^{\urcorner}\right) \rightarrow \mathfrak{t}\right)$ [1, Subst. Equiv.]

3. $B\left(\mathfrak{g},{ }^{\ulcorner} \mathfrak{d} \mathfrak{c}^{\urcorner}\right) \rightarrow\left(B\left(\mathfrak{g},{ }^{\ulcorner} \mathfrak{d} \mathfrak{c}^{\urcorner}\right) \rightarrow \mathfrak{t}\right)$

$\left[2, \wedge^{-}\right]$

4. $B\left(\mathfrak{g},{ }^{\ulcorner} \mathfrak{d} \mathfrak{c}^{\urcorner}\right) \rightarrow \mathfrak{t}$

[3, Contraction]

5. $\mathfrak{d} \mathfrak{c}$ $[1,4, \mathrm{MP}]$

6. $B\left(\mathfrak{g},{ }^{\ulcorner} \mathfrak{d} \mathfrak{c}^{\urcorner}\right)$

[5, Capture]

7. $\mathfrak{t}$ $[4,6, \mathrm{MP}]$

The argument is valid for any arbitrary sentence replacing "God is Brazilian" in the consequent of the divine curry sentence, e.g. absurd sentences like "Grass is blue" and "I am a brain in vat", as well as explosive sentences like "God is Brazilian and God isn't Brazilian" that entail any other sentence. This situation makes $\mathcal{T}$ a trivial theory.

We think that an argument like the Divine Curry makes the problem related to the concept of omniscience even harder to solve. In fact, the purported solutions to paradoxes like the Divine Liar that are based on a reinterpretation of classical negation won't work as a solution to the Divine Curry, because this new paradox doesn't involves essentially the negation as the Divine Liar does 4 Therefore, if we want to change the logical framework

\footnotetext{
${ }^{4}$ On related issues concerning the Liar and Curry paradoxes, see Geach's article On Insolubilia (Geach, 1955).
} 
in order to give an unified response to the paradoxes, it is important to consider alternatives that incorporates an adequate conditional that rejects Contraction and Conditional Proof (CP) used to derive triviality in the the Divine Curry paradox.

\section{A Very Simple Solution}

A very simple solution to the paradoxes is to accept that the sentence $\mathfrak{d} l$ is indeed believed by God, although this simple solution commit us to the claim that God believes contradictions. Cotnoir (Cotnoir, 2017) comes to this startling commitment in his discussion of a member of the Liars' family known as Milne's Paradox. Before we go on the discussion of this commitment, let us first state Milne's paradoxical argument. The starting point of the argument is the self-referential sentence " $N o$ omniscient being knows that which this very sentence expresses.". Let $O$ be the divine omniscience predicate, $\mathfrak{g}$ a term that refer to God and $K$ the knowledge relation between a cognitive agent and a sentence. So we have the self-referential sentence $\lambda$, such that, $\lambda \leftrightarrow \neg \exists x\left(O x \wedge K\left(x,{ }^{r} \lambda^{\urcorner}\right)\right)$, which form the basis of the following argument:

1. $O \mathfrak{g} \wedge K\left(\mathfrak{g},{ }^{\ulcorner} \lambda^{\urcorner}\right)$

2. $K\left(\mathfrak{g},{ }^{\ulcorner} \lambda^{\urcorner}\right)$

3. $\lambda$

4. $\neg \exists x\left(O x \wedge K\left(x,{ }^{\ulcorner} \lambda^{\urcorner}\right)\right)$

5. $\neg\left(O \mathfrak{g} \wedge K\left(\mathfrak{g},{ }^{\ulcorner} \lambda^{\urcorner}\right)\right)$

6. $\left(O \mathfrak{g} \wedge K\left(\mathfrak{g},{ }^{\ulcorner} \lambda^{\urcorner}\right)\right) \rightarrow \neg\left(O \mathfrak{g} \wedge K\left(\mathfrak{g},{ }^{\ulcorner} \lambda^{\urcorner}\right)\right) \quad[1-5, \mathrm{CP}]$

7. $\neg\left(O \mathfrak{g} \wedge K\left(\mathfrak{g},{ }^{\ulcorner} \lambda^{\urcorner}\right)\right)$
[Hypothesis]

$\left[1, \wedge^{-}\right]$

[2,Factivity] 
The first hypothesis says that God is omniscient and knows the sentence $\lambda$. The conclusion is reached by simplification (elimination of conjunction), the factivity of knowledge (a release rule for $\mathrm{K}$ ), the inter-substitution of equivalents based on self-reference, quantifier elimination, Conditional Proof, and Intuitionistic version of Reduction (RAAI). Milne's argument puts a terrible dilemma to theists. They have either to deny omniscience to God or to accept that God at the same time has and doesn't have knowledge of $\lambda 5^{5}$ How can theists avoid this dilemma?

Theists are not prone to take the first alternative and give up the idea of divine omniscience, since omniscience is a divine attribute deeply rooted in the theological tradition and it is displayed in many passages of holy texts like the Bible (e.g. "For he views the ends of the earth and sees everything under the heavens" [Job 28:24]) and the Koran (e.g. "There is nothing whatever like unto Him; and $\mathrm{He}$ is the All-Hearing, the All-Seeing" [Ash-Shura 42:12]). However, it is not easy to accept that God knows contradictions, since accepting contradictions could give rise to some troubling questions. Would we lost the property of factivity of knowledge by attributing to God knowledge of contradictions? Should we also give up the Release rule, since from the fact that God believes that $\alpha$ we could no longer infer that $\alpha$ is the case, since $\alpha$ could also be a false sentence? So far we can see, these are hard questions for which there would be no easy answers.

However, according to Cotnoir, we shouldn't be bothered so much by this dilemma. In fact, his dialetheist response to Milne's paradox is simply to

\footnotetext{
${ }^{5}$ The reason is this: if God is omniscient, he knows all truths, including the truth expressed by $\lambda$, but $\lambda$ just says that no omniscient being knows $\lambda$.
} 
affirm that God could believe contradictions. Moreover, he holds that the dilemma has no devastating power because we surely doesn't loose neither the factivity of knowledge nor the Release rule. He says:

If we allow that God can know contradictions, we allow that God can know some false propositions. This is correct, as far as it goes; but, for the dialetheist, contradictions while being false may also be true. Only contradictions that are true (and false) can be known. Hence, the factivity of knowledge is preserved (Cotnoir, 2017, p. 9)

The reason why the factivity of knowledge and the Release rule are preserved is that from the fact that God believes that $\alpha$ we still get $\alpha$, even though we could also get $\neg \alpha$ if $\alpha$ is a contradictory sentence like Milne's sentence $\lambda$. Perhaps we couldn't always get something like the truth and only the truth, but that doesn't seem to be a big problem and after all "you can't always get what you want".

Cotnoir's dialetheist response to Milne's paradox is very simple indeed, but is it right? In order to accept this response as correct we have to change our definition of omniscience in the following way:

Definition 2. Omniscience*: A being $x$ is omniscient iff for all sentence $\alpha$, (a) $\alpha$ is true iff $x$ believes that $\alpha$, (b) $\alpha$ is false iff $x$ doesn't believe that $\alpha$, except if $\alpha$ is a true sentence as well, (c) if $\alpha$ were true, $x$ would believe that $\alpha$, and finally (d) if $\alpha$ were false, $x$ would not believe that $\alpha$, except if $\alpha$ were a true sentence as well.

In this definition, conditions (a) and (c) perfectly matches respectively the conditions (i) and (iii) of 
our previous definition of Omniscience. Condition (b) matches condition (ii) only partially, since in (b) we introduce the constraint that God believes a false proposition $\alpha$ in $s_{1}$ iff $s_{1}$ is a situation where $\alpha$ is a dialetheia, i.e. a sentence that is not only false, but also true. Likewise, (d) matches partially condition (iv), since in (d) we introduce the constraint that God in $s_{1}$ would believe a false sentence $\alpha$ in a situation $s_{2}$ iff $s_{2}$ were a situation where $\alpha$ is a dialetheia, i.e. a sentence that is not only false, but also true.

This new definition has some remarkable consequences. According to (b), if $\alpha$ is a dialetheia, $\alpha$ is not only true, but also false, that is, $\neg \alpha$ is also true, since the falsehood of a sentence is equivalent to the truth of its negation. Given Capture and Capture* rules, that amounts to say that God believes that $\alpha$ at the same time that he doesn't believe that $\alpha$. That is an absolute weird conclusion, to say the least. But that is not all. A related troublesome consequence of the new definition of omniscience is that we can lose the full trust in God's words if his words are not to be seen as the truth and only the truth. In fact, there is no reason why someone shouldn't refuse God's teachings by alleging that they are false, even though they are also true, and there wouldn't be any reason to disagree with him as well. There wouldn't be also any strong moral compass provided by God's commands if we couldn't see theses commands as absolutely correct, and not correct and false at the same time. How should we act upon contradictory commands? Moreover, the relation between God and human creatures would be severely damaged, since God's promises would always be covered with doubts: Trust the Lord and do good; dwell in the land and enjoy safe pasture. Take delight in the Lord, and he will give you the desires of your 
heart [Psalms 37: 3]. And the Psalm continues scorning the wrongdoers, praising the righteous and promising to them the land where they will dwell forever. Is that the truth and only the truth or this promise could also be false? No one believes that dubious promises could be binding and have enough impact to change the minds and hearts of human beings.

Of course, Cotnoir could improve his response to Milne's paradox. To say that God believes contradictions is not to say that God believes any contradiction. Therefore, the consequences of accepting contradictions wouldn't be so drastic as we have pictured in the last paragraph. Now it would be very important to distinguish the class of contradictions that would be acceptable from those that are not acceptable and this explanation won't be so simple as the first response the dialetheist has given to paradoxes. A further complicating factor is this: even if the dialetheist succeeds in this first task, she will still owe us an explanation about why to distinguish the sets of acceptable contradictions from those that are not acceptable.

It has been a standard view defended in Logic, Metaphysics, and worldly-based semantics that inconsistency implies triviality. In Classical Logic, this view can be expressed by the Principle of Explosion (PE) (or Ex Falso Quodlibet), according to which $\{\alpha, \neg \alpha\} \vDash \beta$ holds for all sentences $\alpha$ and $\beta$, that means, an inconsistent set of sentences logically implies any and every sentence you want. The way that negation and disjunction are classically modelled entails PE ${ }^{6}$ Perhaps, a good reason for $\mathrm{PE}$ is that

\footnotetext{
${ }^{6}$ Let $\alpha$ and $\neg \alpha$ be both true. A disjunction is true iff at least one disjunct is true. Thus $\alpha \vee \beta$ is true. From classical interpretation of negation, $\neg \alpha$ is true iff $\alpha$ is false. Thus $\alpha$ is false and $\alpha \vee \beta$ is true. Therefore, $\beta$ is true. The relevant aspect of negation and disjunction preserved in this proof is also known
} 
the occurrence of true contradictions shouldn't be expected under any theory or assumption, unless we are trying to prove its falsity. After all, contradictions are the most evocative and clear cases of falsity. So if we want to strongly separate truth away from falsehood or trustful from unreliable informants, we should be ready to pay the highest price. That means, we should accept anything to follow from contradictions, and try to avoid them.

Paraconsistency is the most strong reaction against PE. There are many different versions of Paraconsistency, many different philosophical ideas in support of such reaction, and also many different families of logics under this very inclusive brand that don't validate PE. A philosophical motivation for Paraconsistency comes precisely from the alleged existence of true contradictions. For dialetheist:7 the very concept of truth is inconsistent, and that would provide us with many true contradictions or dialetheias, like the paradoxical sentences $\mathfrak{d} \mathfrak{l}$ and $\mathfrak{d} \mathfrak{c}$ and their semantic counterparts $\mathfrak{l}$ and $\mathfrak{c}$. Of course, if the concept of truth is inconsistent, God's omniscience carries the same fate as well. A dialetheist account of truth must provide a paraconsistent logic that not only avoids triviality but also actually solve the aforementioned paradoxes. As we saw, a dialetheist account of $\mathfrak{d} \mathfrak{l}$ and $\mathfrak{d} \mathfrak{c}$ has to face many challenges. An special concern in the present context is that we can not see how any reasonable version of theism can be based on Dialetheism, since it implies that God has not only true beliefs, but also false ones, and so his words could be unreliable at some point.

There seem to be very good reasons to block triviality. Non-triviality provides a minimal criterion

as Disjunctive Syllogism (DS).

${ }^{7}$ See also (Priest, 2006), (Beall, 2009), and (Cotnoir, 2017). 
to separate truth from falsity. There might be many incorrect theories that do not trivialize, but if a theory implies everything, it can not be seriously taken as correct, at least we cannot do this without straightly flirting with Relativism. This is probably one of the strongest reasons against triviality. In this way, except for the relativist, everyone agrees that triviality should be blocked. Nevertheless, Dialetheism is in an awkward position here. If you are a dialetheist, it seems that you have no right to complain about triviality. After all, you are not afraid of contradictions and you are not exactly avoiding falsity. So what makes triviality so scared and dangerous to you? For you, the main important reason against trivialism is lost forever.

Next, we develop a response to paradoxes of omniscience that is also paraconsistent, but unlike Cotnoir's response to Milne's Paradox, it preserves our intuitions about divine omniscience, is not burdened with attributions of contradictory beliefs to God and doesn't give up the main reason for avoiding triviality.

\section{The Logic of Impossible Truths (LIT)}

We offer in this section a new framework for representing God's omniscience and explaining away the paradoxical nature of $\mathfrak{d} \mathfrak{l}$ and $\mathfrak{d} \mathfrak{c}$ sentences and of their counterparts $\mathfrak{l}$ (the Liar sentence) and $\mathfrak{c}$ (the Curry sentence) in traditional semantic paradoxes as well. We name this framework as the Logic of Impossible Truths (LIT). As we are going to show, LIT-models provide not only a more intuitive account for the paradoxical sentences, but they also preserve Transparency of Truth, Capture and Release rules, Factivity and Reliability of God's beliefs, consistency and non-triviality of reality. Besides LIT-models 
provide a non-vacuist interpretation to counterpossibles (counterfactual conditionals with impossible antecedents), which allows us to preserve closely related versions of conditions (iii) and (iv) of Omniscience.

Before proceeding with this presentation of LIT, let us first take an informal look at some aspects of this framework. LIT is a paracomplete paraconsistent logic based on Situation Semantics and a ceteris paribus conditional ${ }^{8}$. LIT-models evaluate sentences in situations in much the same way as worldly-based semantics do, but it doesn't take situations as necessarily complete nor as necessarily consistent. In fact, there are inconsistent situations, although they will never deserve the status of real situations. Conditional and negative sentences will also receive modal treatments, since in order to evaluate them we take account not only of situations in which they hold, but situations in their neighbourhood too.

Situations are the building blocks of our framework. Roughly speaking, situations are ways reality could and could not be. Actual situations are pieces of reality, different parts of reality determined by the insertion of different informational agents in different space-time locations. Informational agents can be differently tuned to the same environment and so be capable of extracting different pieces of information from it. For example, at a meeting to discuss the divine liars and write this article, the authors share the same environment (the same slice of space-time) with Ideiafix, Guilherme's pet dog. By

\footnotetext{
${ }^{8}$ LIT inherits important aspects of Situation Semantics as given by Barwise \& Perry (Barwise \& Perry, 1999), Barwise \& Etchemendy (Barwise \& Etchemendy, 1987), and Mares (Mares, 2004). It also inherits something of the conditional semantics provided by Lewis (Lewis, 1973), Stalnaker (Stalnaker, 1968), and Nolan (Nolan, 2016). Nevertheless, LIT should not be taken to subsume any of those frameworks.
} 
its very accurate sense of smell, Ideiafix can extract pieces of information that we can not extract. On the other side, since we can read English, we can extract pieces of information from the screen of the laptop that Ideiafix can not extract, but only one of us can extract information from the German song playing on the radio.

Accordingly to this example, there are different situations that are at the same time actual, since they are different parts of reality determined by the insertion of different informational agents in different space-time locations. But neither Relativism nor Dialetheism follows from that account. We are not committed to the idea that we live in different worlds, e.g. one world where there is a delicious smell of a rubber ball behind the couch (Ideafix's world) and a totally different world where there are exciting paradoxes to be solved (philosophers' world), nor to the idea that we live in a world with true contradictions, e.g. a world where there is and there is not a delicious smell or an exciting philosophical problem. Actual situations are incomplete, they are determined by different ways in which the same reality is sliced, but there is just one reality.

Moreover, we are not committed to the idea that an informational agent always get things right. We hold that reality can not provide misinformation and some situations are silent about many topics. So, when an informational agent has false beliefs, her false beliefs are not truly based in actual situations, she is basing them somewhere else, namely in some non-actual situation. The non-actual situations are pieces of fictional (alternative) realities. But there are impossible non-actual situations too: non-actual situations can be impossible because we can fictionalize impossible alternatives. For example, a way that reality could not be is the one in which the 
cat in Schrödinger's famous thought-experiment is at the same time dead and alive inside the box. This cat-dead-and-alive situation is a logically impossible (inconsistent) situation, but yet it is an alternative to actual situations that are just silent on the subject. As far we can see, there might be many different sorts of impossibilities (e.g. logical, physical, and metaphysical impossibilities), and LIT-models can be made more accurate in order to distinguish them. For present purposes, however, it suffices to show that there are inconsistent non-trivial situations and that actual situations are free from both contradiction and triviality.

As in worldly-based semantics, We can take situations as classes of sentences, which means, a situation $s$ can be identified with the class of all sentences informed by $s$ (sentences that are true in $s)$. Later, we talk about situations as items of models that include interpretation functions $(\nu)$ that drive us from situations into sets of sentences. Thus the set of sentences we informally associate with $s$ can be precisely identified with $\nu(s)$ under the presumed model.

As we said before, our framework is based on a class of ceteris paribus relations for conditionals. A ceteris paribus relation is always relative to a sentence, it relates a situation to all situations in which such sentence is true and everything else relevant is the same. For example, let $s_{1}$ be a situation in which you find yourself in while reading this article, that is, the set of facts holding around you at a given space-time location. Let us now assume that the sentence "There is intelligent life on other planets" (let's represent it as $\alpha^{9}$ is not true in

\footnotetext{
${ }^{9}$ We use greek small letters for arbitrary formulas, but a more accurate representation for this sentence could be provided with quantifiers and predicate symbols.
} 
$s_{1}$ ( $s_{1}$ doesn't inform you about $\alpha$ ). In the same way, a ceteris paribus relation $R_{\alpha}$ relates $s_{1}$ to all situations where $\alpha$ is true but everything else relevant is the same. If $s_{1} R_{\alpha} s_{2}$ and $s_{1} R_{\alpha} s_{3}$, then $\alpha$ is true in both $s_{2}$ and $s_{3}$. Of course, $s_{1}, s_{2}, s_{3}$ are not exactly the same, but the only difference between them is related to $\alpha$ or something that doesn't matter for the truth of $\alpha$. Maybe in $s_{1}$ you are dressing a blue shirt, in $s_{2}$ a red one, and in $s_{3}$ a black one, for example.

A conditional sentence expresses a general (unspecified) relation of consequence and in that sense it says that the consequent follows from the antecedent (even if the antecedent doesn't happen to be actually true). Thus, in order to evaluate a conditional sentence, we must take into account many different situations in which the antecedent is true. Consider for example the sentence "If there is intelligent life on other planets, we would already know about it". Our present situation doesn't verify nor falsify the antecedent of this conditional, we actually don't know whether there is intelligent life on other planets of our universe. Should we give up the idea of evaluating this conditional? The answer is no! The fact that we don't know whether there is intelligent life on other planets doesn't hinder an intuitive assessment of the conditional itself. It seems that the conditional is false, because it is not only possible but also highly probable that some planets are far enough from Earth to make them entirely (causally, perceptually, and communicatively) isolated from us. Even though we don't have any clues about the existence of intelligent life outside the planet Earth, intelligent unreachable creatures living on planets far away from us is a possible and highly plausible scenario. This scenario falsifies the conditional sentence, showing that the consequent does not follow from the antecedent. 
LIT-models include inconsistent and other sorts of impossible situations and, therefore, ceteris paribus relations might relate possible situations to impossible ones. Consider the conditional sentence "If Schrödinger's cat were both alive and dead inside the box, God wouldn't believe that". Since its antecedent (let us represent it by $\beta$ ) is impossible, it is not only a counterfactual but a counterpossible conditional. According to LIT-models, this conditional is verified in a normal situation $s$ if there is no situation $s_{1}$ such that $s R_{\beta} s_{1}$ and $\neg B\left(\mathfrak{g},{ }^{\ulcorner} \beta^{\urcorner}\right)$is not true in $s_{1}$. In order to $\beta \rightarrow \neg B\left(\mathfrak{g},{ }^{\ulcorner} \beta^{\urcorner}\right)$not to be true in $s$, there must be a situation $s_{1}$ in which $\beta$ is true too. Therefore, there must be an inconsistent situation in order to avoid what has been called Vacuism, the thesis according to which all counterpossibles are vacuoulsy true. As the reader can see with the current example, Vacuism would fight against conditions (iii) and (iv) of Omniscience.

While conditionals are evaluated under ceteris paribus relations, negative sentences should be evaluated under compatibility relation. Compatibility is a relation on situations, it relates all situations that do not contradict each other ${ }^{10}$ Thus $s_{1}$ is incompatible with $s_{2}$ (we write $s_{1} \perp s_{2}$ ) iff $s_{s}$ asserts something that $s_{1}$ denies. $s_{1}$ is compatible with $s_{2}$ $\left(s_{1} C s_{2}\right)$ iff they are not incompatible. That been said, $\neg \gamma$ is true in a situation $s$ iff there is no $s_{1}$, such that, $s C s_{1}$ and $\gamma$ is true in $s_{1}$. Thus a situation informs about the negation of $\gamma$ if it excludes $\gamma$, in the sense that $\gamma$ is true in no situation compatible with such situation 11

\footnotetext{
${ }^{10}$ We are precisely interested here in logical compatibility, but the relation could be redefined in order to grasp different senses of compatibility.

${ }^{11}$ The compatibility negation we are offering here is partially based on Routley semantics as formalized in Restall (Restall, 1999), Beall (Beall, 2009), Mares (Mares, 2004) and others.
} 
As we have already pointed out, LIT-models admit inconsistent situations. Certainly, an inconsistent situation is not compatible with itself (and so the compatibility relation is not reflexive), but that doesn't mean that it is absolutely incompatible. Let $s_{1}=\{\alpha, \neg \alpha, \beta\}, s_{2}=\{\beta, \neg \gamma\}$, and $s_{3}=\{\neg \alpha, \beta\}$. Thus $s_{1} \perp s_{1}, s_{2} C s_{2}, s_{3} C s_{3}, s_{1} C s_{2}$, $s_{2} C s_{3}$, and $s_{1} \perp s_{3}$. When a situation is inconsistent (self-incompatible) about some topic, it is also incompatible with all situations that say something about this topic. But a given situation can be compatible with many situations that remains silent on the topic on which it conflicts with itself. As we have also been emphasizing, many situations are incomplete, in the sense that they remain silent about many topics. The given example with situations $s_{1}, s_{2}$ and $s_{3}$ also shows that compatibility is not a transitive relation. Of course, compatibility is symmetric.

We assume in our framework that for any situation $s$ there is always a maximal-compatible situation with $s$. That means, we add the star operator on situations, such that, for all $s, s_{1}$, if $s C s_{1}$, then $s_{1} \subseteq s^{\star}$. The star operator might be seen as an extremely artificial device of formal semantics. But we think that this is the wrong way to think of situations. We have explained that not all situations are supposed to represent pieces of reality, only the actual ones. As we are going argue, if $s$ is an actual situation, then $s^{\star}$ can not be actual ${ }^{12}$ Despite of that, the point of treating negation as a modal operator is to consider sets of situations for the truth conditions of negative sentences. The star operator on a situation $s$ determines a situation which is maximal-compatible with $s$.

One last remark about the star operator: if $s$ is

${ }^{12}$ This is included ahead in what we call "Star Normality". 
silent about some topic, $s^{\star}$ must contradict itself about that same topic, that means, if $s$ is incomplete, $s^{\star}$ is inconsistent. For example, if neither $\alpha$ nor $\neg \alpha$ is true in $s$, then there are $s_{1}, s_{2}$, such that, $s C s_{1}, s C_{2}, \alpha$ is true in $s_{1}$, and $\neg \alpha$ is true in $s_{2}$. Thus $s_{1} \subseteq s^{\star}, s_{2} \subseteq s^{\star}$, and $s^{\star} \perp s^{\star}$. In the opposite direction now, if $s$ is inconsistent (self-incompatible), $s^{\star}$ is incomplete. In order to see that, let $s$ be such that both $\alpha$ and $\neg \alpha$ are true in $s$. Thus there are no $s_{1}$ such that $s C s_{1}$ and $\alpha$ or $\neg \alpha$ is true in $s_{1}$. Therefore the situation maximalcompatible with $s$ must also remain silent about $\alpha$, neither $\alpha$ nor $\neg \alpha$ is true in $s^{\star}$. Of course, this is not supposed to exclude all contradictions from $s^{\star}$ : if $s$ is also silent about $B$, then even if $s^{\star}$ does not contradict itself about $\alpha$, it surely contradicts itself about $\beta$.

We present below LIT-Language, LIT-models, and logical consequence as defined on LIT-models. We also mention some important properties of LIT and relate them with paradoxical sentences.

Definition 3. Let $\mathcal{L}$ be a formal language based on the following vocabulary: a denumerable set of variables $\left\{v_{0}, v_{1}, v_{2}, \cdots\right\}$, a denumerable set of constants $\left\{k_{0}, k_{1}, k_{2}, \cdots\right\}$, a denumerable set of $n$-place function symbols $\left\{f_{n}^{0}, f_{n}^{1}, f_{n}^{2}, \cdots\right\}$, a denumerable set of $n$-place predicate symbols $\mathbf{P}_{n}=\left\{P_{n}^{0}, P_{n}^{1}, P_{n}^{2}\right\}$, for each natural number $n>0$, the set of logical connectives and quantifiers $\{\neg, \vee, \wedge, \rightarrow, \forall, \exists\}$, and parentheses 13

Some special symbols in the vocabulary of $\mathcal{L}$ need to be stressed since they are going to be interpreted under fixed constraints for LIT-models we present later. Between constants we find a special one for God's name, say $k_{0}$ (we write it as $\mathfrak{g}$ ). Between function symbols we find a one-place naming function

\footnotetext{
${ }^{13} \mathrm{We}$ shall use $x, y, z, \ldots$ for arbitrary variables, $a, b, c, \ldots$ for arbitrary constants, $\alpha, \beta, \gamma, \ldots$ for arbitrary predicates or formulas, $f, g, h, \ldots$ for arbitrary function symbols, and $\Gamma, \Sigma, \Pi$ for arbitrary sets of formulas.
} 
symbol (which gives a name for each formula of $\mathcal{L}$ ), say $f_{0}^{1}$ (we write it as ${ }^{\ulcorner} \ldots{ }^{\urcorner}$). Between predicate letters we find a one-place truth predicate (say $P_{1}^{0}$ ), a one-place omniscience predicate (say $P_{1}^{1}$ ), and a belief two-place predicate, say $P_{2}^{0}$. We write them, respectively, as $T, O$, and $B$.

Definition 4. The set of terms of $\mathcal{L}$ is the least set including all constants, variables, and function symbols applied to terms. A term is closed iff there is no occurrence of a free variable in it. $\mathcal{T}$ is the set of all closed terms.

Definition 5. The set of formulas of $\mathcal{L}$ is the least set $\mathcal{F}$ closed under the following conditions:

(a) If $t_{1}, \ldots, t_{n}$ are terms and $P$ is any $n$-place predicate symbol, $P t_{1}, \ldots, t_{n} \in \mathcal{F}$.

(b) If $\alpha \in \mathcal{F}$, then $\neg \alpha \in \mathcal{F},(\alpha \vee \beta) \in \mathcal{F},(\alpha \wedge \beta) \in \mathcal{F}$, $(\alpha \rightarrow \beta) \in \mathcal{F} 14$

(c) If $\alpha$ is any formula, and $x$ is any variable, $\forall x \alpha(x) \in \mathcal{F}$ and $\exists x \alpha(x) \in \mathcal{F}$.

Definition 6. A sentence is a formula with no occurrence of a free variable in it. The set of sentences is called $\mathcal{S}$.

Next, we define LIT-models for $\mathcal{L}$. Models are constrained by many important conditions we shall present in two different parts, one concerning logical constraints, and the other concerning general constraints on the constitutive relations of models.

Definition 7. A LIT-model for $\mathcal{L}$ is a tuple $\mathcal{M}=\left\langle D, S, N, @,\left\{R_{\alpha} \mid \alpha \in \mathcal{S}\right\}, \nu\right\rangle$ according to logical and general constraints given ahead, where:

${ }^{14}$ As usual, $(\alpha \leftrightarrow \beta)$ can be defined as $(\alpha \rightarrow \beta) \wedge(\beta \rightarrow \alpha)$. 
(a) $D$ is a non-empty class of objects, the domain or universe over which we talk about (on which we interpret language $\mathcal{L}$ ). For each $d \in D$, we add a constant $k_{d}$. Thus, for each model $\mathcal{M}$, we extend $\mathcal{L}$ to $\mathcal{L}_{\mathcal{M}}{ }^{15}$

(b) $S$ is a non-empty class of situations.

(c) $N \subset S$ is a non empty class of normal situations.

(d) @ $\subset S$ is the class of all actual situations in $\mathcal{M}$.

(e) $R_{\alpha}$ is a ceteris paribus accessibility relation for sentence $\alpha$ on situations.

(f) $\nu$ is an interpretation function, such that, it drives us from closed terms of the extended language into the domain $\left(\nu: \mathcal{T}_{\mathcal{L}_{\mathcal{M}}} \longrightarrow D\right)$, it drives us, for each situation $s$, from $n$-place predicates into $n$-tuples of the domain $\left(\nu: S \times \mathbf{P}_{n} \longrightarrow D^{n}\right)$, for all $n \geq 1$, and it drives us from situations into sets of sentences, those sentences that are true in such a situation under such interpretation $(\nu: S \longrightarrow \operatorname{Pow}(\mathcal{S}))$. We add a special notation for "true in situation $s$ under model $\mathcal{M}^{\prime}$, say $\mathcal{M}, s \Vdash \alpha$. Thus $\mathcal{M}, s \Vdash \alpha$ iff $\alpha \in \nu(s)$.

Notice that we opt here for a constant domain through all situations of a given model. However the interpretations of predicates and sentences are relative to situations. This is just a first extricate version of our framework, and we intend in future works to explore LIT-models with variable domains. Notice yet that this simplification do not force all situations to talk about everything inside the domain of the model, since partiality still stands.

\footnotetext{
${ }^{15}$ This is required for adopting a substitutional interpretation of the quantifiers.
} 
It is important to observe that we are including within each LIT-model a set of normal situations. For $\mathcal{M}$ to be a model, it must include situations that obey all logical constraints. There must be situations in which some of these constraints are not forced, but at least some situations are 16 Normal situations are going to be closed under logical consequence, they are the logical situations. Of course, since we are considering also impossible situations, we must consider a radical sort of impossibility, the counterlogical situations, those situations that are not closed under logical consequence (at least they are not closed under logical consequence of LIT). There might be different levels of the impossible, like failure of natural laws, miracles, failure of conceptual laws, contradictions, and failure of the logical laws. Inside LIT-models, contradictions are taken as impossibilities but inconsistent situations can still be closed under the logical consequence provided by LIT. Not all inconsistent situations are counterlogical ones. Nevertheless, we might reason about situations in which even LIT rules fail, these are the non-normal situations.

Given the class of normal situations and the compatibility relation, we can partially define the class of Possible situations $P$. Let $\mathcal{M}$ be a model, $S$ the class of situations of the model, $N \subset S$ the class of normal situations, and $C$ a compatibility relation on $S$. Thus $P \subseteq\{s \in N \mid s C s\}$. Of course if $s \perp s$, $s \notin P$ since consistency is a minimal criteria of possibility. If $s \notin N, s$ is a counterlogical situation, therefore $s \notin P$. Notice that inconsistency does not necessarily imply non-normality nor vice-versa. There might be some weaker levels of impossibility to

\footnotetext{
${ }^{16}$ Notice that there is no circularity in this strategy. It is a commom practice in non-normal modal logics. See chapter 18 of (Priest, 2008), for example.
} 
be distinguished later (maybe in a future work), like situations in which something travels faster then light, the future causes the past, identity fails for persons, and so on. That is the reason why we take $P$ as just a subset of all normal self-compatible situations. This partial definition suffices for our present purposes.

LIT-models include a class of all ceteris paribus relation. For each sentence $\alpha \in \mathcal{S}$, it provides a relation $R_{\alpha} \subseteq S^{2}$. It is more useful to take this relation as a function from situations into classes of situations $\left(f_{\alpha}: S \longrightarrow \operatorname{Pow}(S)\right)$, that means, $f_{\alpha}(s)=\left\{s_{1} \in S \mid s R_{\alpha} s_{1}\right\}$. This function determines, for instance, the class of all situations, ceteris paribus with an actual situation $s$, in which Germany has won the WWII. A different function in the same class of the model delivers the class of situations, ceteris paribus with the actual situation $s$, in which Schrödinger's cat is dead and alive inside the box. So this function relates an actual situation with a class of impossible situations, that means $f_{\gamma \wedge \neg \gamma}(s) \subseteq S-P$, where $S-P$ is the complement of possible situations of the model and $\gamma \wedge \neg \gamma$ is the sentence "Schrödinger's cat is dead and alive inside the box" 17

As a last general remark about LIT-models, consider that worlds can be intuitively understood as complete and consistent situations. Thus $s \in S$ is a world of model $\mathcal{M}$ iff, for all $\alpha \in \mathcal{S}$, either $\alpha$ is true in $s$ or $\neg \alpha$ is true ( $\alpha$ is false) in $s$. However, as we show in Proposition 14 ahead, there are no possible worlds, that means, if $s$ is a world, $s \notin P$ since $s \perp s$, for all $s \in S$ of all models.

Consider now the logical constraints that models are supposed to fit for LIT-models 18

\footnotetext{
${ }^{17}$ We are assuming here that "alive" is equivalent to "not dead". Again, a more complex translation would take place here. This is just a simplification.

${ }^{18}$ Notice that we are just providing constraints for the
} 
Logical Constraints: Let $\mathcal{M}=\left\langle D, S, N\right.$, @, $\left\{R_{\alpha} \mid \alpha \epsilon\right.$ $\mathcal{S}\}, \nu\rangle$ be a LIT-model, $s \in S, s_{1} \in N, \alpha, \beta \in \mathcal{S}, P \in \mathbf{P}_{0}$, and $t_{1}, \ldots, t_{n}$ closed terms in $\mathcal{T}_{\mathcal{L}_{\mathcal{M}}}$. Thus:

1) $\mathcal{M}, s \Vdash P t_{1}, \ldots, t_{n}$ iff $\left\langle\nu\left(t_{1}\right), \ldots, \nu\left(t_{n}\right)\right\rangle \in \nu(P)$.

2) $\mathcal{M}, s \Vdash \neg \alpha$ iff for no $s_{2} \in S$ both $s C s_{2}$ and $\mathcal{M}, s_{2} \Vdash \alpha$.

3) $\mathcal{M}, s \Vdash \alpha \wedge \beta$ iff $\mathcal{M}, s \Vdash \alpha$ and $\mathcal{M}, s \Vdash \beta$.

4) $\mathcal{M}, s \Vdash \alpha \vee \beta$ iff $\mathcal{M}, s \Vdash \alpha$ or $\mathcal{M}, s \Vdash \beta$.

5) $\mathcal{M}, s_{1} \Vdash \alpha \rightarrow \beta$ iff $f_{\alpha}\left(s_{1}\right) \subseteq[\beta] 19$

6) $\mathcal{M}, s \Vdash \forall x \alpha(x)$ iff $\mathcal{M}, s \Vdash \alpha(x / t)$, for all $t \in$ $\mathcal{T}_{\mathcal{L}_{\mathcal{M}}} 20$

7) $\mathcal{M}, s \Vdash \exists x \alpha(x)$ iff $\mathcal{M}, s \Vdash \alpha(x / t)$, for some $t \epsilon$ $\mathcal{T}_{\mathcal{L}_{\mathcal{M}}}$

Conjunction and disjunction behaves in a pretty classical way pace the aforementioned fact that LIT-models include both incomplete and inconsistent situations. We've already made the relevant comments about logical constraints about conditional and negative sentences. Still it is important to notice that all situations are closed under logical constraints for conjunction, disjunction, negation, and quantifiers, but only normal situations are closed under conditional constraints. There might be different levels of the impossible, but for now we are interested in paradoxical sentences like $\mathfrak{l}, \mathfrak{d} \mathfrak{l}, \mathfrak{c}$ and $\mathfrak{d} \mathfrak{c}$ and inconsistency and triviality are the only relevant types of impossibility that concern us. Since

functions $\nu$ of LIT-models. Clause $f$ of definition 7 already adds $\Vdash$.

${ }^{19}$ Where $[\beta]=\{s \mid \mathcal{M}, s \Vdash \beta\}$.

${ }^{20}$ Where $\alpha(x / t)$ is the result of replacing all free ocurances of $x$ in $\alpha$ with $t$. 
conditional sentences allows us to (non-vacuously) reason about the impossible, the conditional constraint should be restricted to normal situations; even if we reason about counterlogical, our reasoning is supposed to be fixed by our own logic (otherwise, it wouldn't be really reasoning, right?).

Next we provide a list of general constraints for LIT-models that concern self-reference, compatibility relation, truth, omniscience, and ceteris paribus relations:

General Constraints: Let $\mathcal{M}=\left\langle D, S, N\right.$, @, $\left\{R_{\alpha} \mid\right.$ $\alpha \in \mathcal{S}\}, \nu\rangle$ be a LIT-model, $s, s_{1} \in S, \alpha \in \mathcal{S}$, and $\beta \in \mathcal{F}$ any formula with a free occurrence of $x$. Thus: 21

8) there is a sentence $\gamma$, such that, $s \Vdash \gamma$ iff $s \Vdash$ $\beta\left({ }^{r} \gamma^{\urcorner}\right)$(for all $\left.s \in N\right)$.

[Self-Reference]

9) If $s, s_{1} \in @$, then $s, s_{1} \in N$, and $s C s_{1}$.

[Non-Dialetheism]

10) There is $s^{\star}$, such that, $s C s^{\star}$ and $s_{2} \subseteq s^{\star}$, for every $s_{2}$, such that, $s C s_{2}$.

[Star Postulate]

11) $s^{\star \star}=s$

[Double Star]

12) $f_{\alpha}(s) \subseteq[\alpha]$, for all $s \in N$.

[Identity]

13) If $s \Vdash \alpha$, then $s \in f_{\alpha}(s)$, for all $s \in N$.

[Weak Centering]

14) If $s \in N$, then $s^{\star} \in S-N$. If $s \in S-N$, then $s^{\star} \in N$.

[Star Normality]

15) $\nu(s, T) \subseteq \mathcal{S}$, and if $s \in N, s \Vdash T\left({ }^{\ulcorner} \alpha^{\urcorner}\right)$iff $s \Vdash \alpha$.

[Transparency]

\footnotetext{
${ }^{21}$ From now on we ommit any explicit mention to models when they can be unambiguously determined by the context.
} 
16) $\nu(s, B) \subseteq(D-\mathcal{S}) \times \mathcal{S}$, for some $s_{2} \in @, s_{2}, \Vdash O(\mathfrak{g})$, and, for all $s \in N$, if $s \Vdash O(\mathfrak{g})$, then $s \Vdash \alpha$ iff $s \Vdash B\left(\mathfrak{g},{ }^{\ulcorner} \alpha^{\urcorner}\right)$.

[Omniscience]

Constraint 8 is introduced for allowing self-reference to play the game of paradoxes in LIT-models. As it is usually emphasized, natural languages provide many different sources for self-reference, e.g. indexicals, definite descriptions, and empirical circumstances ${ }^{22}$ In formal contexts, self-reference is usually delivered through arithmetization techniques, which is basically the essence behind the Diagonal Lemma. Here we accept that all normal situations include self-referential sentences like $\mathfrak{l}, \mathfrak{d} \mathfrak{l}, \mathfrak{c}$, and $\mathfrak{d} \mathfrak{c}$. In fact, we are pushing a stronger version of self-reference, since we are not expressing it by means of a biconditional, but as logical equivalence. Thus since $\neg T(x) \in \mathcal{F}$, there is a sentence $\mathfrak{l}$ (the Liar Sentence), such that, for all $s \in N, s \Vdash \mathfrak{l}$ iff $s \Vdash \neg T\left({ }^{\ulcorner} \mathfrak{l}\right)$ (for any model $\mathcal{M}$ ).

Constraint 9 is Non-Dialetheism. LIT is an alternative framework to Dialetheism, a paraconsistent non-dialetheist logic, designed for representing omniscience of God and transparency of truth. Thus we restrict models to those in which all actual situations are normal and self-compatible (consistent). We take Non-Dialetheism for granted, but we argue next that LIT-models provide intuitive answers to paradoxes and besides they are better ways for representing transparency of truth and omniscience.

Constraints 10 and 11 are the necessary constraints for LIT-negation to be as strong as FDE-negation. Because of 10 and 11, Double Negation and De Morgan rules hold in LIT. As we said before, we assume here that, for all $s \in S$, there

\footnotetext{
${ }^{22}$ See Kripke (Kripke, 1975).
} 
is a a situation $s^{\star}$ that is the maximal-compatible situation with $s$. We also assume that $s^{\star \star}=s$, that means, the maximal-compatible situation with $s^{\star}$ is $s$ itself. Notice yet that if $s C s$, then $s \subseteq s^{\star}$ (for all $s \in S)$.

Constraints 12 and 13 are related to conditional sentences. Models restricted as in 12 are those in which Identity $(\alpha \rightarrow \alpha)$ holds in all normal situations. Models restricted as in 13 are those closed under Modus Ponens (MP). Notice that 12 and 13 are both intuitive contraints on LIT models. 12 says that if $s R_{\alpha} s_{1}$, then $s_{1} \Vdash \alpha$, that means, a ceteris paribus situation in which $\alpha$ is true is a situation in which $\alpha$ is true. 13 says that if $s \Vdash \alpha$, then $s$ is also a situation ceteris paribus in which $\alpha$ is true. A stronger version of 13 would say that if $s \Vdash \alpha, s$ would be the only one situation ceteris paribus with $s$ in which $\alpha$ is true. This would make sense if we were modelling similarity relations, but for now we are not concerned about that kind of relations. So Weak Centering suffices for our purposes.

Constraint 14 is about the relation between maximal-compatible situations and normal situations. The main point here is that the Star Operator jumps from side to side between normal and non-normal situations. Consider for example the empty situation $s_{\varnothing}\left(\right.$ such that $\left.\nu\left(s_{\varnothing}\right)=\varnothing\right)$ and the full situation $s_{\mathcal{S}}\left(\right.$ such that $\left.\nu\left(s_{\mathcal{S}}\right)=\mathcal{S}\right)$. On the one hand, as we are going to prove below (Proposition 11, item 1), for all $s \in N$ and all $\alpha \in \mathcal{S}$ of all models, $s \Vdash \alpha \rightarrow \alpha$ (Identity). Thus $s_{\varnothing} \notin N$. On the other, $s_{\mathcal{S}}$ is closed under all logical constraints. In fact, $s_{\mathcal{S}}$ is an explosive (trivial) situation, but yet $s_{\mathcal{S}} \in N$. It is important to note that $s_{\varnothing}^{\star}=s_{\mathcal{S}}$ and $s_{\mathcal{S}}^{\star}=s_{\varnothing}$. The existence of the empty situation $s_{\varnothing}$ is then required for Star Operator as the maximal-compatible situation with $s_{\mathcal{S}}$, the full, trivial and explosive 
situation. Moreover, since nothing is true in $s_{\varnothing}$, this situation is not closed under logical consequence and, therefore, $s_{\varnothing} \in S-N$.

Constraint 15 is for Transparency of truth. The first conjunct of 15 says that in all situations of all models $T$ is a predicate of sentences. The second conjunct says that it is a transparent predicate in all normal situations.

Constraint 16 is for God's Omniscience. First it says that $B$ is a relation between (non-sentences) things and sentences. Second it establishes that at least some actual situation informs about God's Omniscience. Third it says that if $s$ informs about God's omniscience ( $s$ is a God-situation) then $B(\mathfrak{g}, x)$ is like a truth transparent predicate in $s$. We are taking Theism for granted as much in the same way as we are taking Non-Dialetheism for granted. We offer LIT-models as an alternative framework for God's Omniscience, one non-dialetheist version of Theism that deals with the aforementioned paradoxes.

Transparency of Truth is preserved in LIT. Constraint 15 gives us all versions of Capture and Release for truth in all normal situations (including the negative-clauses with *). Capture and Release versions of God's omniscience, however, hold only for situations which inform God's Omniscience. But the reader might be worried: if $s \mathbb{H} B\left(\mathfrak{g},{ }^{\ulcorner} \alpha^{\urcorner}\right)$for some actual $s$ of some model and $\alpha$ true in $s$, does it means that we have an atheist model? There is no reason to worry. It makes no difference if $s \in$ @ or not (and neither if $s \in P$ or not), since what " $\left.s \Vdash B\left(\mathfrak{g},{ }^{r} \alpha\right\urcorner\right)$ " tells us is that some situation doesn't inform God's Omniscience. We would have an atheist model if some actual $s$ informed about God's ignorance, but this will never happen, since such models are forbidden: all models include actual situations that 
inform God's Omniscience. Notice that we also need some non-actual situations in order to present models for sentences like "If God didn't believe that, then it would be false.". As we are going to see in the next section (Proposition 20), we can also preserve Capture and Release rules for models.

With models restricted as in definition 5 with Logical and General Constraints, we can define logical consequence (validity) as preservation of truth in all normal situations of all models 23

Definition 8. Let $\Gamma \subseteq \mathcal{S}$ and $\alpha \in \mathcal{S}$. Thus, $\Gamma \vDash \alpha$ iff there is no model $\mathcal{M}=\left\langle @, N, S,\left\{R_{\alpha} \mid \alpha \in \mathcal{S}\right\}, \nu\right\rangle$ and $s \in N$, such that, $\mathcal{M}, s \Vdash \Gamma$ and $\mathcal{M}, s \Vdash \alpha{ }^{24}$

We show now some important properties of LIT and relate them with paradoxical sentences.

\section{Proposition 9.}

1. LIT is paracomplete. $\not \neq \alpha \vee \neg \alpha$

2. LIT is paraconsistent. $\alpha \wedge \neg \alpha \not \beta$.

Proof. Let $\mathcal{M}$ be a model with $S=\left\{s, s_{1}, s^{\star}, s_{1}^{\star}\right\}$, and $\alpha, \beta \in \mathcal{S}$, such that, $s, s_{1} \in N, s C s_{1}, s \Vdash \alpha, s \Vdash \neg \alpha$, $s \Vdash \beta, s_{1} \Vdash \alpha, s_{1} \Vdash \neg \alpha$, and $s_{1} \Vdash \beta$. Excluded Middle fails in $s$, Explosion fails in $s_{1}$.

Proposition 10. Let $\mathcal{M}$ be a model with $s \in S$, and $\alpha \in \mathcal{S}$. Thus:

1. $s \Vdash \neg \neg \alpha$ iff $s^{\star} \Vdash \alpha$.

2. $s \Vdash \alpha$ iff $s^{\star} \Vdash \neg \alpha$.

\footnotetext{
${ }^{23}$ As usual, tautologies are special cases of validity, those formulas that follow from $\varnothing$.

${ }^{24} \mathcal{M}, s \Vdash \Gamma$ sse $\mathcal{M}, s \Vdash \beta$, for all $\beta \in \Gamma$.
} 
Proof. Item 1: Let $s \Vdash \neg \alpha$. From Star Postulate, and negation constraint, then $s C s^{\star}$, and $s^{\star} \Vdash \alpha$. Now let $s^{\star} \Vdash \alpha$. From Star Postulate again, $s_{1} \Vdash \alpha$ (for all $s_{1}$, such that, $s C s_{1}$ ). From negation constraint, $s \Vdash \neg \alpha$. Item 2: Let $s \Vdash \alpha$. From Star Postulate, and negation constraint, $s^{\star} \Vdash \neg \alpha$. Now let $s^{\star} \Vdash \neg \alpha$. From prop. 10.1, $s^{\star \star} \Vdash \alpha$, but $s^{\star \star}=s$ (Double Star).

\section{Proposition 11.}

1. $\vDash \alpha \rightarrow \alpha$.

2. $\alpha, \alpha \rightarrow \beta \vDash \beta$.

3. $\neg \neg \alpha=\vDash \alpha$.

4. $\neg \alpha \wedge \neg \beta=\neg(\alpha \vee \beta)$.

5. $\neg(\alpha \wedge \beta) \Rightarrow \vDash \neg \alpha \vee \neg \beta$.
[Identity]

[Modus Ponens]

[Double Negation]

[De Morgan]

[De Morgan]

Proof. Item 1: It follows from Identity constraint (13) and the logical constraint of the conditional (5). Item 2: Let $\mathcal{M}$ be a model with $s \in N$, such that, $s \Vdash$ $\alpha, s \Vdash \alpha \rightarrow \beta$. Thus $f_{\alpha}(s) \subseteq[\beta]$ (logical constraint of the conditional), and $s \in f_{\alpha}(s)$ (Weak Centering). Therefore, $s \Vdash \beta$. Item 3: Let $\mathcal{M}$ be a model with $s \in N$, and $s \Vdash \neg \neg \alpha$. From prop. 10, it follows that $s^{\star} \Vdash \neg \alpha$, and $s \Vdash \alpha$. Now let $s \in N$, and $s \Vdash \alpha$. From prop. 10, $s^{\star} \Vdash \neg \alpha$, and $s^{\star \star} \Vdash \alpha$, but $s^{\star \star}=s$ (Double Star). Item 4: Let $\mathcal{M}$ be a model with $s \in N, s \Vdash \neg \alpha$, and $s \Vdash \neg \beta$. Suppose (for reduction) that $s \Vdash \neg(\alpha \vee \beta)$. Thus, $s^{\star} \Vdash \alpha \vee \beta$, contradicting hypothesis. Now let $s \in N$ and $s \Vdash \neg(\alpha \vee \beta)$. Thus $s^{\star} \Vdash(\alpha \vee \beta)$. Therefore, $s \Vdash \neg \alpha$, and $s \Vdash \neg \beta$. Item 5: Let $\mathcal{M}$ be a model with $s \in N$, and $s \Vdash \neg(\alpha \wedge \beta)$. Thus $s^{\star} \Vdash \alpha$ or $s^{\star} \Vdash \beta$. Therefore, $s \Vdash \neg \alpha \vee \neg \beta$. Now let $s \in N$, and $s \Vdash \neg \alpha$ or $s \Vdash \neg \beta$. Thus $s^{\star} \Vdash(\alpha \wedge \beta)$, and $s \Vdash \neg(\alpha \wedge \beta)$. 


\section{Proposition 12.}

1. $\not \beta \beta \rightarrow(\alpha \rightarrow \alpha)$.

2. $\alpha \rightarrow \beta, \neg \beta \not \neg \alpha$.

3. $\neg \alpha, \alpha \vee \beta \not \beta$.

4. $\alpha \rightarrow(\alpha \rightarrow \beta) \not \vDash \alpha \rightarrow \beta$.

5. $\Gamma, \alpha \vDash \beta$ does not imply $\Gamma \vDash \alpha \rightarrow \beta$.

6. $\alpha \rightarrow \neg \alpha \not \neg \alpha$.
[Relevant Identity]

[Modus Tollens]

[Disjunctive Syllogism]

[Contraction]

[CP]

[RAAI]

Proof. Item 1: Consider a model with situations $s, s_{1}$ such that $s \in N, s_{1} \in f_{\beta}(s)$ (thus $s_{1} \Vdash \beta$ ), but $s_{1} \Vdash \alpha \rightarrow$ $\alpha$. Of course, $s_{1} \in S-N$. Still, $s \Vdash \beta \rightarrow(\alpha \rightarrow \alpha)$. Item 2: Consider a model with $s \in N, f_{\alpha}(s) \subseteq[\beta], s \Vdash \neg \beta$, but $s \Vdash \neg \alpha$. Thus $s^{\star} \Vdash \alpha$, and $s^{\star} \Vdash \beta$. Notice that if $s C s$, and $s^{\star} \in N$, this model would not be available, but Star Normality assures us that $s^{\star} \in S-N$. Item 3: Consider a model with $s \in N, s \Vdash \alpha \wedge \neg \alpha$, but $s \| \forall$. Of course, in this case, $s \perp s$, but this is not a problem. Item 4: Consider a model with $s \in N$, $f_{\alpha}(s)=\left\{s_{1}\right\}, s_{1} \Vdash \alpha, s_{1} \Vdash \alpha \rightarrow \beta$, but $s_{1} \in S-N$, and $s_{1} \Vdash \beta$. Thus $f_{\alpha}(s) \subseteq[\alpha \rightarrow \beta]$, but $f_{\alpha}(s) \nsubseteq[\beta]$. Item 5: $\alpha \vDash \beta \rightarrow \beta$ (from item 1 of prop. 11). Now item 1 of prop. 12 provides a counter-example to item 5 of 12 . Thus there is a model with $s \in N$ such that $f_{\alpha}(s) \nsubseteq[\beta \rightarrow \beta]$, and for some $s_{1} \in f_{\alpha}(s), s_{1} \Vdash \beta \rightarrow \beta$. Therefore $f_{\alpha}(s) \cap(S-N) \neq \varnothing$. Item 6: Consider a model with $s \in N, f_{\alpha}(s) \subseteq[\neg \alpha]$, but $s \Vdash \neg \alpha$. Since $s \in$ $N$, and so it is closed under the consequence relation, we have to conclude also that $s \Vdash \alpha$.

\section{Paradoxes Again}

In section 4 we introduced LIT language, models, and proved some important features of this logic. Now we 
finally use this framework to obtain the sentences $\mathfrak{l}, \mathfrak{d} \mathfrak{l}$, $\mathfrak{c}, \mathfrak{d} \mathfrak{c}$, and we show important results in LIT that are related to them.

Proposition 13. Let $\mathcal{M}$ be a model with $s \in N$, and $\mathfrak{t}$ a triviality constant sentence. Thus:

1. There is a sentence $\mathfrak{l}$, such that, $s \Vdash \mathfrak{l}$ iff $s \Vdash$ $\neg T\left({ }^{\ulcorner} \mathfrak{l}\right)$.

2. There is a sentence $\mathfrak{d} l$, such that, $s \Vdash \mathfrak{d} l$ iff $s \Vdash$ $\neg B\left(\mathfrak{g},{ }^{\ulcorner} \mathfrak{d} \mathfrak{l}^{\urcorner}\right)$.

[Divine Liar]

3. There is a sentence $\mathfrak{c}$, such that, $s \Vdash \mathfrak{c}$ iff $s \Vdash$ $T\left({ }^{r} \mathfrak{c}^{\urcorner}\right) \rightarrow \mathfrak{t}$.

[Curry]

4. There is a sentence $\mathfrak{d} \mathfrak{c}$, such that, $s \Vdash \mathfrak{d} \mathfrak{c}$ iff $s \Vdash$ $B\left(\mathfrak{g},{ }^{\ulcorner} \mathfrak{d} \mathfrak{c}^{\urcorner}\right) \rightarrow \mathfrak{t}$.

[Divine Curry]

Proof. 1-4 follow from straight applications of self-reference constraint on the following formulas: 1 . $\neg T(x), 2 . \neg B(\mathfrak{g}, x), 3 . T(x) \rightarrow \mathfrak{t}$, and $4 . B(\mathfrak{g}, x) \rightarrow \mathfrak{t}$.

In cases 3 and $4, \mathfrak{t}$ is a constant that represents triviality (explosion). In fact, we have a curry sentence and a divine curry sentence for each sentence of the language. Dealing with sentences where $\mathfrak{t}$ takes the consequent position is just a simplification. Let us see now what are the consequences of these sentences from the point of view of LIT-models.

Proposition 14. Let $\mathcal{M}$ be a model with $s \in S$. Thus:

1. If $s \in N$, and $s C s$, then $s \Vdash \mathfrak{l}$ and $s \Vdash \neg \mathfrak{l}$.

2. If $s \in N$, and $s C s$, then $s^{\star} \perp s^{\star}$.

3. If $s$ is a world, then $s \notin P$. 
Proof. Item 1: From prop. 13, and Transparency, if either $s \Vdash \mathfrak{l}$ or $s \Vdash \neg \mathfrak{l}$, then $s \perp s$ (by MP). Therefore, if $s \in N$, and $s C s$, then $s \Vdash \mathfrak{l}$ and $s \Vdash \neg \mathfrak{l}$ Item 2: It follows from prop. 14.1, and prop. 10. Item 3: Since worlds are complete situations, if $s$ is a world, either $s \Vdash \mathfrak{l}$ or $s \Vdash \neg l$. From prop. 14.1, if $s \in N, s \perp s$, therefore $s \notin P$. If $s \in S-N$, by definition $s \notin P$.

Proposition 15. Let $\mathcal{M}$ be a model with $s \in S$. Thus:

1. If $s \in @$, then $s \Vdash \mathfrak{d} \mathfrak{l}$ and $s \Vdash \neg \mathfrak{d} l$.

2. If $s \in @, s$ is not a world.

Proof. Item 1: From Omniscience (constraint 16), there is an $s_{1} \in$ @, such that, $s_{1} \Vdash$ ol iff $s_{1} \Vdash B\left(\mathfrak{g},{ }^{\mathfrak{r}} \mathfrak{l} \mathfrak{l}\right)$. From prop.13, $s \Vdash \quad \mathfrak{d} l$ iff $s \Vdash \neg B\left(\mathfrak{g},{ }^{\mathfrak{d}} \mathfrak{d} \mathfrak{l}\right)$ (all $\left.s \in N\right)$. Thus if $s \in$ @, and either $s \Vdash \mathfrak{d} \mathfrak{l}$ or $s \Vdash \neg \mathfrak{d l}$, there is an $s_{2}$ such that $\nu\left(s_{2}\right)=\nu(s) \cup \nu\left(s_{1}\right)$, and $s_{2} \in @$, but $s_{2} \perp s_{2}$, contradicting constraint 9 (Non-Dialetheism). Item 2: From definition of worlds and proposition 14.1 (or 15.1).

Proposition 16. Let $\mathcal{M}$ be a model with $s \in N$, and $s \neq s_{\mathcal{S}}$. Thus $s \Vdash \mathfrak{c}$.

Proof. From proposition 13, if $s \in N, s \Vdash \mathfrak{c}$ iff $s \Vdash$ $T\left({ }^{r} \mathfrak{c}^{\urcorner}\right) \rightarrow \mathfrak{t}$. From Transparency, $s \Vdash \mathfrak{c}$ iff $s \Vdash T\left({ }^{\ulcorner} \mathfrak{c}^{\urcorner}\right)$. Thus, from MP, if $s \Vdash \mathfrak{c}$ then $s \Vdash \mathfrak{t}$, i.e. everything is true in $s$. Therefore, there is only one normal situation in which $\mathfrak{c}$ is true, the explosive situation $s_{\mathcal{S}}$.

Notice that if $s \in N$, and $s \Vdash \mathfrak{c}$, then $f_{T\left({ }^{r} \mathfrak{c}^{\urcorner}\right)}(s) \nsubseteq[\mathfrak{t}]$. Therefore, there is $s_{1} \in f_{T\left({ }^{\prime} \mathfrak{c}^{\prime}\right)}(s)-[\mathfrak{t}]$. In this case, for such model to obey the aforementioned constraints, if $s_{1} \neq s_{\mathcal{S}}$, then $s_{1} \notin N$. What this shows is that for 
Curry sentences not to be true in a normal situation $s$, there must be a non-normal one $s_{1}$ where the sentence $\mathfrak{c}$ is true, but the consequent $\mathfrak{t}$ is not (a non-explosive non-normal situation).

Proposition 17. Let $\mathcal{M}$ be a model with $s \in @$. Thus $s \Vdash \mathfrak{d} \mathfrak{c}$.

Proof. According to Omniscience, there is $s_{1} \in @$, such that, $s_{1} \Vdash \mathfrak{d} \mathfrak{c}$ iff $s_{1} \Vdash B\left(\mathfrak{g},{ }^{\mathfrak{d}} \mathfrak{c} \mathfrak{c}\right)$. For all $s \in N, s \Vdash \mathfrak{d} \mathfrak{c}$ iff $s \Vdash B\left(\mathfrak{g},{ }^{\mathfrak{d}} \mathfrak{c} \mathfrak{c}^{\urcorner}\right) \rightarrow \mathfrak{t}$. Thus if $s \in @$ and $s \Vdash \mathfrak{d} \mathfrak{c}$, there is $s_{2} \in @$, such that, $\nu\left(s_{2}\right)=\nu(s) \cup \nu\left(s_{1}\right)$, and $s_{2} \Vdash \mathfrak{t}$ (from two applications of MP), contradicting Non-Dialetheism.

Next we add some new definitions concerning models in order to provide stronger results about paradoxical sentences:

Definition 18. Let $\mathcal{M}$ be a model and $\alpha \in \mathcal{S}$. Thus:

1. $\alpha$ is true in $\mathcal{M}$ iff there is some $s \in @$ such that $s \Vdash \alpha$.

2. $\alpha$ is false in $\mathcal{M}$ iff $\neg \alpha$ is true in $\mathcal{M}$.

3. God believes $\alpha$ in $\mathcal{M}$ iff some $s \in @$ is such that $s \Vdash B\left(\mathfrak{g},{ }^{\ulcorner} \alpha^{\urcorner}\right)$.

4. God disbelieves $\alpha$ in $\mathcal{M}$ iff some $s \in @$ is such that $s \Vdash \neg B\left(\mathfrak{g},{ }^{\ulcorner} \alpha^{\urcorner}\right)$.

Proposition 19. Let $\mathcal{M}$ be any model. Thus:

1. $\mathfrak{l}$ is neither true nor false in $\mathcal{M}$.

2. $\mathfrak{d} l$ is neither true nor false in $\mathcal{M}$.

3. $\mathfrak{c}$ is not true in $\mathcal{M}$.

4. $\mathfrak{d} \mathfrak{c}$ is not true in $\mathcal{M}$. 
Proof. It follows from definition 18, the assumption that actual situations are normal self-compatible (NonDialetheism), and propositions 14-17.

Proposition 20. Let $\mathcal{M}$ be a model. Thus:

1. God believes all (and only the) truths in $\mathcal{M}$.

2. God believes no falsity in $\mathcal{M}$.

Proof. It follows from definition 18 and the fact that $\left.s \Vdash \neg B\left(\mathfrak{g},{ }^{\ulcorner} \alpha\right\urcorner\right)$ iff $s \Vdash B\left(\mathfrak{g},{ }^{\ulcorner} \neg \alpha^{\urcorner}\right)$, for all $s \in N$ of all models.

Proposition 20 captures condition (i) and (ii) of definition of Omniscience provided in section 1. More importantly, it avoids the problem we raised against Dialetheism, namely the unreliability of God's beliefs. Since no contradiction is true in actual situations of models, God believes all and only actual truths, and God disbelieve all and only actual falsities, the unreliability problem does not show up against LIT-models.

LIT-models also capture closely related versions of conditions (iii) and (iv) of the aforementioned definition. As we saw, some actual situations inform about God's Omniscience. A non-actual situation $s$ might include non-actual truths and might include even an inconsistent truth in it, but if this were a God-situation, it would also be true that in $s$ God believes an inconsistent truth. Consider another example. Call cat the the cat-dead-and-alive situation from Schrödinger thought-experiment. Let $\gamma \wedge \neg \gamma$ be the sentence "the cat is dead and alive inside the box". Let cat be a God-situation. Thus cat $\notin P$ since cat $\perp$ cat. Nevertheless cat $\Vdash B\left(\mathfrak{g},{ }^{\ulcorner} \gamma \wedge \neg \gamma^{\urcorner}\right)$. 
It seems that unless non-normal situations are included under $f_{\alpha}(s)$, when $s$ is an actual God-situation, all instances of the schema $\alpha \rightarrow B\left(\mathfrak{g},{ }^{r} \alpha^{\urcorner}\right)$are going to be true in $s$ (the same is valid for $\left.\neg \alpha \rightarrow \neg B\left(\mathfrak{g},{ }^{\ulcorner} \alpha^{\urcorner}\right)\right)$. So God not only believes all actual truths, and disbelieves all actual falsities, but he would also believe alternative truths and disbelieve alternative falsities whereas such alternatives keep things inside normal consequences preserving God's omniscience.

This is an interesting result: if Schrödinger's cat were alive and dead inside the box, God would believe that. And this is even more interesting: if the Liar sentence were true, God would believe that. Both results are far from Dialetheism, since we are not saying that God actually believes the Liar, but that he would believe the Liar is telling the truth, even if what is told is an impossible truth. We take this result to get as close as it could reasonably be of conditions (iii) and (iv) of omniscience.

Before leaving this section, let us just say a few words about the results about paradoxical sentences that we proved in it. These propositions show that LIT provides a paracomplete paraconsistent framework in which both Transparency and Omniscience hold without contradiction. With a modal interpretation of both negation and conditional, we can preserve the consistency of actual situations by giving up their completeness. What does the incompleteness of actual situations mean? It means that completeness is not consistent with Transparency of truth and Omniscience of God. We believe that this idea of completeness being inconsistent with Transparency and Omniscience is somehow shared by many different theories. Dialetheism is precisely a case in point, but dialetheists preserve completeness by giving up 
consistency. Grim, on the opposite side of the debate, choose consistency and pay the price of incompleteness, but since he takes the concepts of Truth and divine omniscience to be inherently complete, he gives them up. Accordingly to him, truths and worlds are always incomplete, their extensions can never be taken as complete totalities. So there is no such thing as Truth nor an omniscient being like God. When we look at the results provided by LIT, we see a path-way between Scylla and Charybdis.

We agree with Grim that to intend to wrap reality and truth as a whole is naive and wrong. Grim provides an ingenious argument against the existence of the set of all truths ${ }^{25}$ Let $T=\left\{t_{1}, t_{2}, t_{3}, \cdots\right\}$ be the set of all truths. Thus for each $\Sigma \in \operatorname{Pow}(T)$, there is a truth about $\Sigma$, for example, $t_{1} \notin \varnothing, t_{1} \in\left\{t_{1}\right\}$, $t_{1} \in\left\{t_{1}, t_{2}\right\}$, etc. Thus there are as many truths as subsets of $T$, contradicting Cantor's Theorem according to which $|T|<\left.|\operatorname{Pow}(T)|\right|^{26} \quad$ Grim is convinced that truth is an indefinite extensible concept, one for which we can not consistently give a complete extension. We agree. That result is behind the aforementioned result in our framework. Of course, Grim's argument demands a lot in order to be formalized. It requires at lest a language in which we can talk about sets of sentences and do Set Theory. Now his argument can not be formalized in our framework, for instance. Nevertheless, we think that the incompleteness of actual situations in our framework mirrors his views about the indefinite extensibility of Truth and Reality. In LIT-models, all

\footnotetext{
${ }^{25}$ See (Grim, 1991), chapter 4.

${ }^{26}$ Using Cantor's diagonalization procedure, Grim shows that the assumption that $\mathrm{T}$ and $\operatorname{Pow}(\mathrm{T})$ are equivalent leads to contradiction. So there cannot be the set of all truths. That's really a very ingenious argument, also used by him in order to prove that there can be no set of all worlds.
} 
worlds are inconsistent. This is supposed to be understood as showing that worlds are (with all non-actual situations) just fictions. Whenever we think and talk about all-inclusive situations we fictionalize about completeness, since there can be no actual complete situation.

We agree with Grim on many points, but we don't share his views on divine omniscience. Omniscience is well represented in LIT-models, despite its paracompleteness. Even if there is no such thing as a complete actual situation (a world), there is no reason to maintain that God is an impossible being. As already pointed out, if some actual $s$ does not inform about God's Omniscience, this doesn't imply that God is not omniscient. For the same reason, an actual situation carrying information that God is omniscient is not supposed to carry all truths about reality as a whole. Remember that a situation is determined by the insertion of an informational agent in a space-time location. So we might not be able to express God's point of view in our framework, that means, to represent a situation provided by the insertion of an universal informational agent in a universal space-time location. But we are able to express God's omniscience in all partial slices of reality.

\section{Final Remarks}

We purpose LIT-models as an alternative framework to represent God's Omniscience and Truth Transparency. In this article we have mainly focused on the motivations behind LIT and on the paradoxical sentences. Of course, there are many more different concerns to be addressed about this framework that we couldn't exhaust in this article. We mention next some of them. 
A very common and strong objection against paracomplete approaches to the Liar Paradox is the Problem of Revenge. Whatever the way you phrase paracompleteness (truth value gaps or third indeterminate values, for instance), it seems that we always leave open the road for stronger versions, if the language is strong enough to represent how we evaluate (or how we don't evaluate) the paradoxical sentences. This is also known as the Hydra like nature of the paradoxes of self-reference, in the sense that every time you cut off one of their menacing heads, two even more menacing ones appear in the same place. The important question to be answered here is whether LIT-models leave open the route for Revenge. We plan a future article to deal precisely with this question.

As the reader surely have noticed, LIT language also doesn't have the usual alethic modal operators for Necessity and Possibility. The concepts of Necessity and Possibility are crucial to our work, but we are neither defining nor axiomatizing them. The reason why we frame LIT-models with situations is that we understand conditional and negation as modal operators as well. It is surely important, in a future work, to relate usual accessibility relation with compatibility and ceteris paribus relation. This would allow us to extend LIT language with alethic modal operators like $\square$ and $\diamond$. This would be the focus of a second future work extending ideas in the present article.

As we mentioned in section 1 , there are many different versions of paradoxes and arguments of impossibility against Theism based on the omni-attributes of God, like omnipotence and omnibenevolence. Omnipotence, for instance, gives rise to the well-known Paradox of the Stone. We believe these arguments of impossibility share the 
same assumption about completeness and consistency so deeply enlightened in LIT-models. Thus we also plan a future work applying LIT-models for different versions of arguments of impossibility.

\section{References}

BARWISE, J. and ETCHEMENDY, J. The Liar: An essay on truth and circularity. OUP. New York. 1987.

BARWISE, J. and PERRY, J. Situations and Attitudes. CSLI Publications 1999.

BEALL, JC. Spandrels of Truth. Oxfod. 2009.

BERTO, F., FRENCH, R., PRIEST, G., and RIPLEY, D. Williamson on Counterpossibles. Journal of Philosophical Logic, 47, pp 693-713. 2018. DOI: $10.1007 / \mathrm{s} 11245-016-9424-\mathrm{x}$

CRAIG, E. Knowledge and the State of Nature. Oxford: Clarendon Press, 1990

COTNOIR, A.J. Theism and Dialetheism. Australasian Journal of Philosophy, 2017. DOI: 10.1080/00048402.2017.1384846

GEACH, P. On Insolubilia. Analysis, Volume 15, Issue 3, January 1955. DOI:10.1093/analys/15.3.71

KRIPKE, S. Outline of a Theory of Truth. In Journal of Philosophy, volume 72, pp. 690-716. 1975. DOI: $10.2307 / 2024634$

GRIM, P. The Incomplete Universe. Cambridge: MIT Press, 1991

LEWIS, D. Counterfactuals. Oxford 1973. 
MARES, E. Relevant Logic: A Philosophical Interpretation. Cambridge, 2004.

NOLAN, D. Conditionals and Curry. Philosophical Studies, vol. 173(10). 2016. DOI: $10.1007 / \mathrm{s} 11098-016-0666-7$.

PRIEST, G. In Contradiction. OUP. Oxford. 2006.

PRIEST, G. An Introduction to Non-Classical Logic: From if to is. Cambridge University Press. New York. 2008.

RESTALL, G. Negation in relevant logics (How I stopped worrying and learned to love the Routley star). In D. M. Gabbay and H. Wansing, editors, What is Negation?, Applied Logic Series. Springer, 1999.

STALNAKER, R. A theory of conditionals. Studies in logical theory. American Philosophical Monograph Series, vol. 2, pp.98-112. 1968.

\section{(c) BY}

